\title{
Real-World Outcomes of Gemcitabine, Cisplatin, and Nab-Paclitaxel Chemotherapy Regimen for Advanced Biliary Tract Cancer: A Propensity Score-Matched Analysis
}

\author{
Kwangrok Jung, Jaewoo Park, Jae Hyup Jung, Jong-Chan Lee, Jaihwan Kim, and Jin-Hyeok Hwang \\ Department of Internal Medicine, Seoul National University Bundang Hospital, Seoul National University College of Medicine, \\ Seongnam, Korea
}

\section{Article Info}

Received July 26, 2021

Revised September 30, 2021

Accepted October 29, 2021

Published online January 7, 2022

Corresponding Author

Jin-Hyeok Hwang

ORCID https://orcid.org/0000-0002-5643-8461

E-mail woltoong@snu.ac.kr

\begin{abstract}
Background/Aims: Advanced biliary tract cancer (BTC) is associated with poor survival. A recent phase II study of triplet combination chemotherapy, including gemcitabine, cisplatin, and nanoparticle albumin-bound (nab)-paclitaxel, has shown promising results. This study aimed to compare the efficacy of triplet and standard doublet chemotherapy in a real-world setting.

Methods: Patients with advanced BTC treated with triplet and doublet chemotherapy regimens were recruited. The propensity-score nearest neighbor matching method with a ratio of oneto-one was used to create a matched cohort for comparison. Progression-free survival (PFS), overall survival (OS), and safety profiles were examined in both groups.

Results: A total of 68 patients ( $n=34$ per group) were included in the matched cohort, and their baseline characteristics were well balanced. Survival outcomes in the triplet chemotherapy group were not better than those in the doublet chemotherapy group, with a median PFS of 7.5 months (95\% confidence interval [Cl], 4.1 to 10.9$)$ versus 7.2 months $(95 \% \mathrm{Cl}, 5.6$ to 8.9$)$ (hazard ratio [HR], $0.93 ; 95 \% \mathrm{Cl}, 0.53$ to $1.62 ; \mathrm{p}=0.793)$ and a median OS of 13.7 months ( $95 \% \mathrm{Cl}, 8.8$ to 18.7$)$ versus 12.2 months ( $95 \% \mathrm{Cl}, 8.4$ to 16.0 ) (HR $0.73 ; 95 \% \mathrm{Cl}, 0.38$ to $1.41 ; \mathrm{p}=0.354$ ), respectively. In addition, the treatment-related severe adverse events, such as neutropenia, were more common in the triplet chemotherapy group.

Conclusions: Gemcitabine, cisplatin, and nab-paclitaxel did not improve the PFS or OS compared to that achieved by standard chemotherapy in patients with advanced BTC. The benefits of triplet chemotherapy in advanced BTC require examination in large randomized controlled trials. (Gut Liver 2022;16:798-805)
\end{abstract}

Key Words: Biliary tract cancer; Gemcitabine; Cisplatin; Albumin-bound paclitaxel

\section{INTRODUCTION}

Biliary tract cancer (BTC) is a group of heterogeneous diseases that originate from the bile duct and gallbladder and includes intrahepatic cholangiocarcinoma (IHCC), extrahepatic cholangiocarcinoma (EHCC), and gallbladder cancer. ${ }^{1}$ BTC is rare and accounts for less than $1 \%$ of global cancer cases; however, its incidence varies worldwide and has increased over the past four decades. ${ }^{2-5}$ The prognosis of BTC is poor, with a 5 -year survival rate of $<20 \%{ }^{6}$ Surgical resection is the only potentially curative option for
BTC; however, nearly two-thirds of patients are diagnosed at an advanced stage with inoperable disease; more than half of patients that undergo surgery experience recurrence. $^{7-10}$

The gemcitabine and cisplatin doublet chemotherapy has been a first-line treatment for BTC for over 10 years. ${ }^{11}$ However, even with this potent doublet chemotherapy, the median overall survival (OS) remains $<1$ year. ${ }^{12,13}$ Other chemotherapy combination regimens have been examined as candidate treatments that may improve survival outcomes in patients with advanced BTC. ${ }^{14-17}$ Among them, 
triplet chemotherapy regimen that includes gemcitabine, cisplatin, and nanoparticle albumin-bound (nab)-paclitaxel has shown promising results in a phase II study with the median progression-free survival (PFS) of 11.8 months and the median OS of 19.2 months. ${ }^{18}$ Consequently, the triplet chemotherapy regimen is currently being used in clinical practice and a phase III trial of this regimen is ongoing (NCT03768414).

Nevertheless, real-world patient outcomes have not been compared between the doublet and triplet chemotherapy regimens to date. The present study aimed to show the efficacy and safety profiles of triplet chemotherapy in a real-world setting and compare them with those of the current standard doublet chemotherapy.

\section{MATERIALS AND METHODS}

\section{Patients}

We identified 41 patients diagnosed with advanced BTC (histologically confirmed IHCC, EHCC, and gallbladder cancer) from September 1, 2019, to December 31, 2020, who received triplet chemotherapy at the Seoul National University Bundang Hospital, Seongnam, Korea. Among them, those who had undergone prior palliative chemotherapy or were lost to follow-up before the first response evaluation were excluded. Finally, 34 patients were included in the triplet chemotherapy group. In addition, we reviewed medical records of patients with advanced BTC who received doublet chemotherapy as first-line chemotherapy between January 1, 2011, and December 31, 2018. The total number of advanced BTC patients treated with doublet chemotherapy was 261 .

We performed propensity-score matching analysis with the nearest neighbor matching method, using the ratio of one-to-one for two different chemotherapy groups matched on age, sex, tumor location, stage, Eastern Cooperative Oncology Group performance status, and carbohydrate antigen 19-9 (CA19-9) to overcome the heterogeneity of baseline characteristics. Finally, a matched cohort of 68 patients ( $\mathrm{n}=34$ patients per group) was obtained for further analyses. This study was approved by the Institutional Review Board of the Seoul National University Bundang Hospital (IRB number: L-2021-642) and informed consent was waived due to study design.

\section{Treatment regimens}

In the doublet chemotherapy group, patients received gemcitabine of $1,000 \mathrm{mg} / \mathrm{m}^{2}$ and cisplatin of $25 \mathrm{mg} / \mathrm{m}^{2}$ as a standard dose on days 1 and 8 every 21 days. ${ }^{11}$ Patients in the triplet chemotherapy group were treated with reduced- dose regimen of $800 \mathrm{mg} / \mathrm{m}^{2}$ gemcitabine, $25 \mathrm{mg} / \mathrm{m}^{2}$ cisplatin, and $100 \mathrm{mg} / \mathrm{m}^{2}$ nab-paclitaxel, as a standard dose on days 1 and 8 every 21 days. ${ }^{18}$ Dose modification or interruption were at the discretion of the attending physician. Granulocyte colony stimulating factors (GCSF) were administered therapeutically or prophylactically at the attending physician's discretion.

\section{Endpoints and assessment}

The primary endpoints were PFS and OS of the triplet and doublet chemotherapy groups. Treatment responses were evaluated continuously at the intervals of 2 or 3 months with computed tomography or magnetic resonance imaging, according to the Response Evaluation Criteria in Solid Tumors, version 1.1. The median follow-up duration was evaluated in all patients including those who died; the median follow-up duration, PFS, and OS were calculated from the day of chemotherapy initiation. When a patient subsequently underwent surgery or radiotherapy, the median follow-up duration and PFS were measured until the time of surgery or radiotherapy.

The secondary endpoints were overall response rate (ORR) and disease control rate (DCR) in both groups. In addition, the median number of treatment cycles and the mean relative dose intensity were calculated to confirm that each chemotherapy agent was suitably administered. Lastly, safety profiles including the rates of hematologic adverse events, non-hematologic adverse events, and use of GCSF prophylaxis were reviewed in both groups. Adverse events were monitored at every hospital visit and graded according to the National Cancer Institute Common Terminology Criteria for Adverse Events, version 4.0.

\section{Statistical analysis}

Continuous variables were analyzed using the t-test, paired t-test, or Mann-Whitney U test, depending on whether the assumption of normal distribution was satisfied or not. Categorical variables were compared with the McNemar test, chi-square test, or Fisher exact test. The Kaplan-Meier curves and Cox proportional hazard models were used to perform survival analysis. All statistical analyses were performed using SPSS version 25.0 (IBM Corp, Armonk, NY, USA).

\section{RESULTS}

\section{Baseline characteristics}

A total of 34 patients in each of the triplet and doublet chemotherapy groups were matched (Fig. 1). Before propensity-score matching, there were differences between 


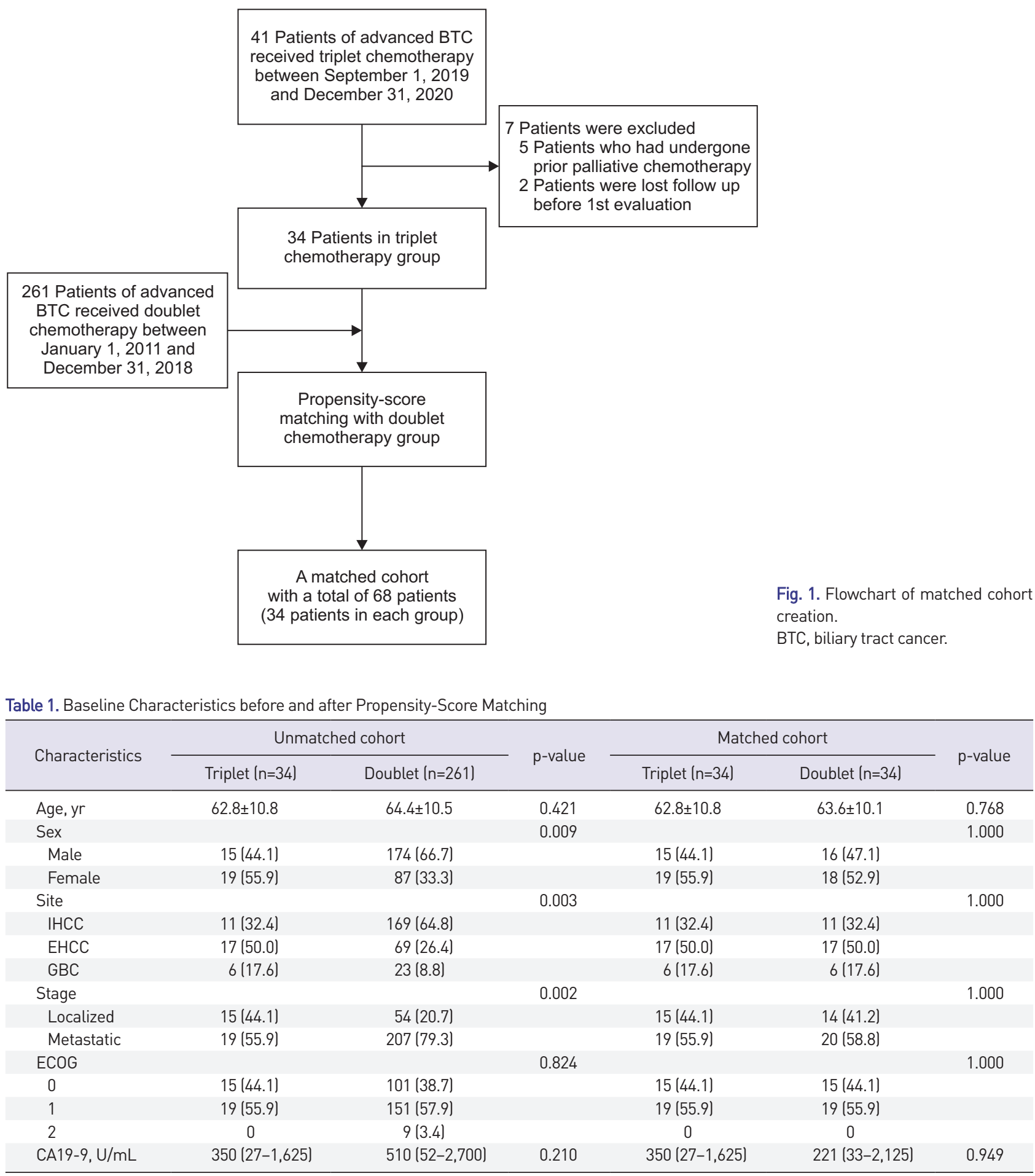

Data are presented as mean \pm SD, number (\%), or median (interquartile range).

IHCC, intrahepatic cholangiocarcinoma; EHCC, extrahepatic cholangiocarcinoma; GBC, gallbladder cancer; ECOG, Eastern Cooperative Oncology Group performance status; CA19-9, carbohydrate antigen 19-9.

the groups in several baseline variables; the baseline characteristics became balanced after matching (Table 1). In the matched cohort, the mean age (standard deviation) in the triplet and doublet groups was $62.8( \pm 10.8)$ and $63.6( \pm 10.1)$ years, respectively. There were more female than male patients in both groups (19/34 [55.9\%] vs 18/34 [52.9\%], respectively). The most common tumor location was EHCC (17/34 per group, 50.0\%), and metastatic cancer was more common than locally advanced cancer in both groups (19/34 [55.9\%] vs 20/34 [58.8\%], respectively). 
The median CA19-9 level (interquartile range) was 350 $(27-1,625) \mathrm{U} / \mathrm{mL}$ in the triplet chemotherapy group and $221(33-2,125) \mathrm{U} / \mathrm{mL}$ in the doublet chemotherapy group.

\section{Efficacy}

During the median follow-up duration (standard deviation) of 8.7 months (95\% confidence interval [CI], 6.3 to $11.2)$ in the triplet chemotherapy group, 21 of 34 patients (61.8\%) experienced disease progression, three patients
(8.8\%) underwent surgery, and two patients (5.9\%) received radiotherapy. In the doublet chemotherapy group, 33 of 34 patients (97.1\%) experienced disease progression or death, except for one patient who underwent surgery.

The median PFS was 7.5 months (95\% CI, 4.1 to 10.9 ) in the triplet chemotherapy group and 7.2 months (95\% CI, 5.6 to 8.9 ) in the doublet chemotherapy group (hazard ratio, 0.93 ; $95 \% \mathrm{CI}, 0.53$ to $1.62 ; \mathrm{p}=0.793$ ) (Fig. $2 \mathrm{~A}$ ). The median OS estimates in the triplet and doublet groups
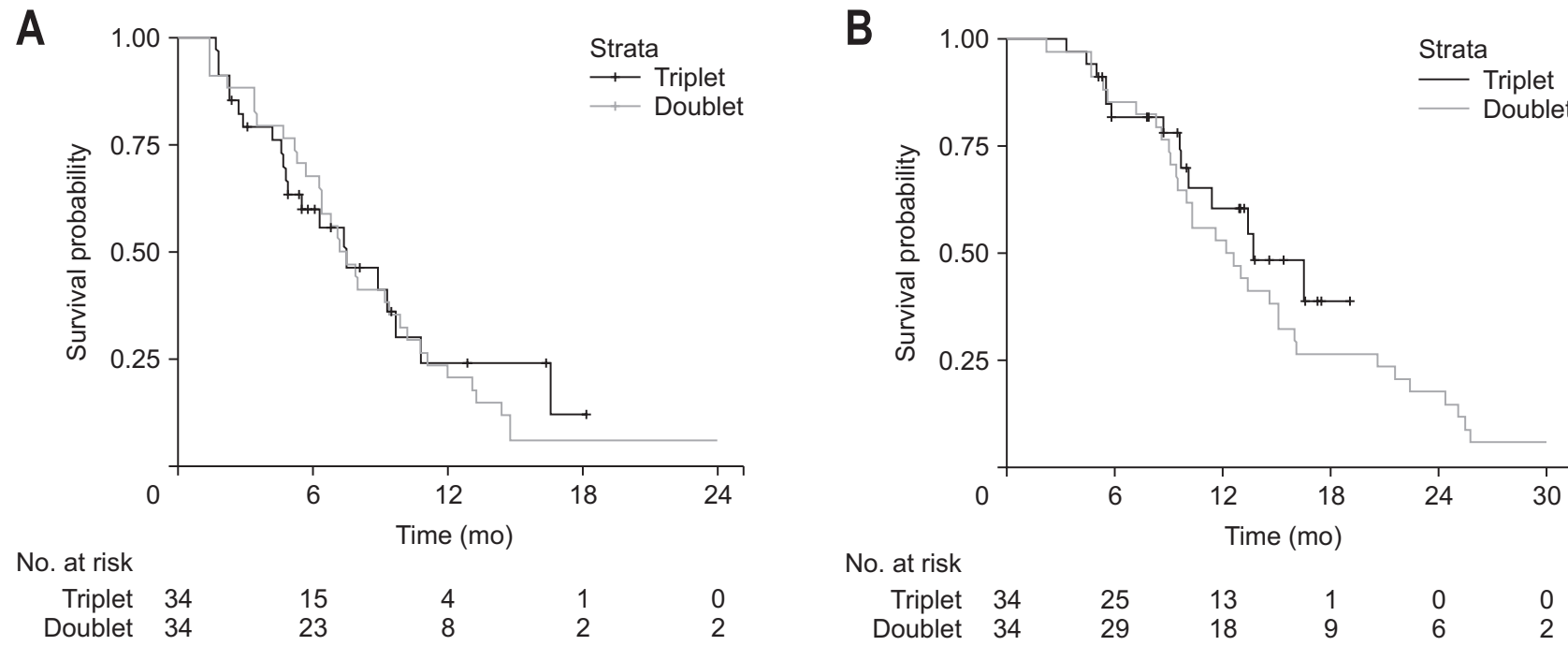

Fig. 2. Kaplan-Meier curves for median progression-free survival and overall survival. (A) The median progression-free survival was 7.5 months in the triplet chemotherapy group and 7.2 months in the doublet chemotherapy group (hazard ratio, $0.93 ; 95 \%$ confidence interval, 0.53 to 1.62 ; $\mathrm{p}=0.793$ ). (B) The median overall survival was 13.7 months in the triplet chemotherapy group and 12.2 months in the doublet chemotherapy group (hazard ratio, $0.73 ; 95 \%$ confidence interval, 0.38 to $1.41 ; p=0.354$ ).

Table 2. Multivariate Analysis of Progression-Free Survival and Overall Survival

\begin{tabular}{|c|c|c|c|c|}
\hline \multirow{2}{*}{ Prognostic factors } & \multicolumn{2}{|c|}{ Progression-free survival } & \multicolumn{2}{|c|}{ Overall survival } \\
\hline & $\operatorname{HR}(95 \% \mathrm{Cl})$ & $\mathrm{p}$-value & $\operatorname{HR}(95 \% \mathrm{Cl})$ & $\mathrm{p}$-value \\
\hline Triplet vs doublet & $1.06(0.55-2.04)$ & 0.857 & $0.70(0.33-1.48)$ & 0.351 \\
\hline Age & $1.01(0.97-1.06)$ & 0.519 & $1.00(0.95-1.05)$ & 0.963 \\
\hline \multicolumn{5}{|l|}{ Sex } \\
\hline Male & Reference & & Reference & \\
\hline Female & $1.58(0.76-3.32)$ & 0.224 & $1.27(0.58-2.78)$ & 0.548 \\
\hline \multicolumn{5}{|l|}{ Tumor site } \\
\hline $\mathrm{IHCC}$ & Reference & & Reference & \\
\hline $\mathrm{EHCC}$ & $1.50(0.70-3.21)$ & 0.294 & $0.87(0.38-2.00)$ & 0.743 \\
\hline GBC & $0.94(0.39-2.41)$ & 0.955 & $1.13(0.44-2.92)$ & 0.803 \\
\hline \multicolumn{5}{|l|}{ Stage } \\
\hline Localized & Reference & & Reference & \\
\hline Metastatic & $3.29(1.63-6.64)$ & 0.001 & $2.70(1.27-5.76)$ & 0.010 \\
\hline \multicolumn{5}{|l|}{ ECOG } \\
\hline 0 & Reference & & Reference & \\
\hline 1 & $1.10(0.50-2.43)$ & 0.819 & $1.06(0.44-2.56)$ & 0.905 \\
\hline \multicolumn{5}{|l|}{ CA19-9 } \\
\hline$<300 \mathrm{U} / \mathrm{mL}$ & Reference & & Reference & \\
\hline$\geq 300 \mathrm{U} / \mathrm{mL}$ & $1.29(0.66-2.54)$ & 0.458 & $1.24(0.63-2.43)$ & 0.540 \\
\hline
\end{tabular}

$\mathrm{HR}$, hazard ratio; $\mathrm{Cl}$, confidence interval; IHCC, intrahepatic cholangiocarcinoma; EHCC, extrahepatic cholangiocarcinoma; GBC, gallbladder cancer; ECOG, Eastern Cooperative Oncology Group performance status; CA19-9, carbohydrate antigen 19-9. 
were 13.7 months (95\% CI, 8.8 to 18.7 ) and 12.2 months (95\% CI, 8.4 to 16.0 ), respectively (hazard ratio, 0.73 ; $95 \%$ CI, 0.38 to $1.41 ; \mathrm{p}=0.354$ ) (Fig. $2 \mathrm{~B}$ ). The median PFS and OS in the triplet chemotherapy group did not show any significant improvement, as compared with those in the doublet chemotherapy group. Adjustment for other baseline variables did not alter these outcomes (Table 2).

In the triplet chemotherapy group, one patient showed complete response and nine patients showed partial response, yielding the ORR of $29.4 \%$ (Table 3 ). The DCR for the triplet chemotherapy group was 79.4\%, including 16 patients with stable disease. In the doublet chemotherapy group, nine patients showed partial response, accounting for the ORR of $26.5 \%$. The DCR was $85.3 \%$ with additional 20 patients who had stable disease. There was no significant difference in ORR and DCR between two groups.

\section{Dose administration and adverse events}

The median number of treatment cycles in the triplet group and doublet group was 6 (interquartile range, 3 to 12) and 8 (interquartile range 4 to 13 ), respectively (Table 4). The mean relative dose intensity of each chemotherapy agent in triplet chemotherapy group was comparable to doublet group (gemcitabine of $85.2 \%$, cisplatin of $81.1 \%$, and nab-paclitaxel of $73.4 \%$ in triplet group vs gemcitabine of $84.1 \%$ and cisplatin of $79.1 \%$ in doublet group). Seven patients $(20.6 \%)$ in triplet group had to discontinue at least one chemotherapy agent due to adverse events (nabpaclitaxel of six patients and cisplatin of one patient), but three patients $(8.8 \%)$ in doublet group (cisplatin of three patients).

The most frequent treatment-related adverse events in both groups were hematologic adverse events. Grade 3 or higher hematologic adverse events were more frequent in the triplet chemotherapy group than in the doublet chemotherapy group (neutropenia rates of $29.4 \%$ vs $20.6 \%$; febrile neutropenia rates of $14.7 \%$ vs $8.8 \%$; anemia rates of $26.5 \%$ vs $20.6 \%$; thrombocytopenia rates of $20.6 \%$ vs $17.6 \%$, respectively) (Table 5). In particular, neutropenia was relatively common in the triplet chemotherapy group, despite six patients (17.6\%) in this group having received prophylactic GCSF and no one in doublet chemotherapy group.

\section{DISCUSSION}

The present study compared the efficacy of a triplet chemotherapy regimen (gemcitabine, cisplatin, and nabpaclitaxel) with that of a doublet chemotherapy regimen (gemcitabine and cisplatin) in the treatment of advanced BTC. Contrary to expectations, in the present study, survival outcomes associated with the triplet chemotherapy (median PFS of 7.5 months, median OS of 13.4 months, and ORR of $29.4 \%$ ) were not superior to those associated with the doublet chemotherapy.

In the present doublet chemotherapy group, the median PFS was 7.2 months, median OS was 12.2 months, and ORR was $26.5 \%$. These findings were comparable to those of the ABC-02 study, in which the corresponding outcomes for the doublet chemotherapy group were 8.0 months, 11.7 months, and $26.1 \%$, respectively. ${ }^{11}$

However, the efficacy of the triplet chemotherapy regimen in this study was inferior to that of previous phase

Table 3. Response to Treatment in the Matched Cohort

\begin{tabular}{lccc}
\hline \multicolumn{1}{c}{ Response } & Triplet $(\mathrm{n}=34)$ & Doublet $(\mathrm{n}=34)$ & $\mathrm{p}$-value \\
\hline Complete response & $1(2.9)$ & 0 & - \\
Partial response & $9(26.5)$ & $9(26.5)$ & - \\
Stable disease & $17(50.0)$ & $20(58.8)$ & - \\
Progressive disease & $7(20.6)$ & $5(14.7)$ & - \\
Overall response & $10(29.4)$ & $9(26.5)$ & 0.595 \\
Disease control & $27(79.4)$ & $29(85.3)$ & 0.525 \\
\hline
\end{tabular}

Data are presented as number $(\%)$.

Table 4. Administration of Chemotherapy Agents in Both Groups

\begin{tabular}{lccc}
\hline Administration & Triplet & Doublet & p-value \\
\hline Treatment cycle & 6 (3-12) & $8(4-13)$ & 0.416 \\
Relative dose intensity, \% & & & \\
Gemcitabine & $85.2 \pm 15.0$ & $84.1 \pm 11.7$ & 0.733 \\
Cisplatin & $81.1 \pm 16.3$ & $79.1 \pm 17.5$ & 0.636 \\
Nab-paclitaxel & $73.4 \pm 21.5$ & - & - \\
\hline
\end{tabular}

Data are presented as median (interquartile range) or mean \pm SD 
Table 5. Safety Profiles in the Matched Cohort

\begin{tabular}{|c|c|c|c|}
\hline Safety profiles & Triplet (n=34) & Doublet (n=34) & $p$-value \\
\hline Any grade $\geq 3 \mathrm{AEs}$ & $20(58.8)$ & 14 (41.2) & 0.146 \\
\hline \multicolumn{4}{|l|}{ Grade $\geq 3$ hematologic AEs } \\
\hline Neutropenia & $10(29.4)$ & $7(20.6)$ & 0.401 \\
\hline Anemia & $9(26.5)$ & $7(20.6)$ & 0.567 \\
\hline Thrombocytopenia & $7(20.6)$ & $6(17.6)$ & 0.758 \\
\hline Febrile neutropenia & $5(14.7)$ & $3(8.8)$ & 0.452 \\
\hline Prophylactic GCSF use & $6(17.6)$ & 0 & 0.025 \\
\hline \multicolumn{4}{|c|}{ Grade $\geq 3$ non-hematologic AEs } \\
\hline Infection & 2 (5.9) & 2 (5.9) & 1.000 \\
\hline Diarrhea & 2 (5.9) & 0 & 0.493 \\
\hline Constipation & $1(2.9)$ & $1(2.9)$ & 1.000 \\
\hline Nausea & $1(2.9)$ & $3(8.8)$ & 0.614 \\
\hline Vomiting & $1(2.9)$ & $1(2.9)$ & 1.000 \\
\hline Rash & $1(2.9)$ & 0 & 1.000 \\
\hline Liver dysfunction & $1(2.9)$ & 0 & 1.000 \\
\hline Renal dysfunction & 0 & 0 & - \\
\hline Neuropathy & $2(5.9)$ & $1(2.9)$ & 1.000 \\
\hline Thromboembolic event & $1(2.9)$ & 0 & 1.000 \\
\hline
\end{tabular}

Data are presented as number (\%).

AE, adverse event; GCSF, granulocyte colony stimulating factors.

II study with triplet chemotherapy regimen (median PFS of 11.8 months, median OS of 19.2 months, and ORR of $45.1 \%) .{ }^{18}$ This discrepancy may be due to the differences in the participants' baseline characteristics. In this study, patients were older (mean age 62.8 years vs 58.4 years in the previous study), the most common tumor location was EHCC $(17 / 34,50.0 \%)$ in contrast to IHCC $(38 / 60,63 \%)$ in the previous study, and the median CA19-9 level was higher $\left(350 \mathrm{U} / \mathrm{mL}\right.$ vs $99 \mathrm{U} / \mathrm{mL}$ in the previous study). ${ }^{18}$ Age and CA19-9 levels are well-documents prognostic factors; ${ }^{19,20}$ nevertheless, differences in prognoses according to tumor location remain controversial. ${ }^{13,21,22}$ In a large retrospective study with 740 advanced BTC patients treated with the doublet chemotherapy, tumor location did not affect survival outcomes. ${ }^{13}$ However, another collective study with individual data from ABC trials showed that IHCC was associated with relatively better OS. ${ }^{21}$ Therefore, considering the effect of potential prognostic factors, we performed a propensity-score matching study showing no difference in survival outcomes between the triplet and doublet chemotherapy groups, even though the doublet chemotherapy group showed outcomes comparable to those reported in the ABC-02 study. ${ }^{11}$

In the present study, the triplet chemotherapy group was initially treated with a reduced-dose regimen, which was recommended in the previous phase II trial. Given that only 28 of 60 patients (46.7\%) in the previous study received the reduced-dose regimen and the ORR in reduceddose group was lower than that in the high-dose group in the previous study $(39.1 \%$ vs $50.0 \%)$, ${ }^{18}$ a reduced-dose may be associated with poor outcomes in the triplet chemotherapy group of this study. However, the reduced-dose group in the previous study included only $10(35.7 \%)$ progression or mortality events, obscuring efficacy outcomes of the reduced-dose regimen. Further large-scale studies are required to confirm the efficacy and safety profiles of the reduced-dose regimen.

In addition, the differences in efficacy outcomes may result from genomic diversity of BTC patients. Several genomic studies in BTC have shown that the molecular profile of BTC varies depending on its anatomical location and underlying etiology. ${ }^{23}$ Moreover, the prognosis may differ according to specific genetic alterations such as KRAS or TP53, or clusters by mutational signature..$^{23-25}$ These genomic findings may help account for the differences in BTC outcomes, which seem to depend on multiple factors such as tumor location, risk factor or etiology, and genetic predisposition. ${ }^{23-26}$ Consequently, further studies on the clinical outcomes of BTC require models that account for diverse tumor biology.

In the present study, hematologic adverse events were the most common type of treatment-related adverse events in both groups. Meanwhile, grade 3 or higher hematologic adverse events, in particular, neutropenia, were more frequent in the triplet chemotherapy group than in the doublet chemotherapy group. The safety profile of the triplet chemotherapy regimen, including hematologic and nonhematologic adverse events, in this study was similar to that in the previous phase II study, except that severe neutropenia was less common in this study $(29.4 \%$ vs $40.4 \%){ }^{18}$ 
However, it should be noted that $30 \%$ of the patients experienced grade 3 or higher neutropenia, even though all patients in the present triplet chemotherapy group received a reduced-dose regimen and $18 \%$ of the patients received prophylactic GCSF.

Our study has several limitations. First, the number of patients treated with triplet chemotherapy was small because this regimen is not yet recommended as standard treatment for advanced BTC. Second, this was a retrospective study, so there was no standardized protocol in the treatment process such as dose modification or GCSF administration. However, chemotherapy administration in both groups, as expressed by the median number of treatment cycles and the mean relative dose intensity, were similar enough to compare the efficacy of two different chemotherapy regimens. Lastly, follow-up duration of patients in triplet chemotherapy group was relatively short. However, the triplet chemotherapy group $(\mathrm{n}=34)$ included $21(61.8 \%)$ patients with disease progression event, five (14.7\%) patients with another subsequent treatment such as surgery or radiotherapy who could not provide additional survival information associated with triplet chemotherapy, and five (14.7\%) patients with a longer follow-up duration than the median PFS. Consequently, these data were considered sufficient for the assessment of survival outcomes in the triplet chemotherapy group.

Our study has some strengths. First, to the best of our knowledge, this is the first study on the new triplet chemotherapy regimen in a real-world setting; in addition, this is the first study to compare patient outcomes between the triplet and doublet regimens. Although this was a retrospective study, the potential confounding factors were well balanced in both groups by propensity-score matching.

In conclusion, the use of gemcitabine, cisplatin, and nab-paclitaxel in patients with advanced BTC did not show survival outcomes better than those associated with the use of gemcitabine and cisplatin, which is the current standard regimen. Moreover, treatment-related severe adverse events, such as neutropenia, were more common in the triplet chemotherapy group than in the doublet chemotherapy group. The use of the triplet chemotherapy in clinical practice requires further evidence from a phase III trial.

\section{CONFLICT OF INTEREST}

No potential conflict of interest relevant to this article was reported.

\section{AUTHOR CONTRIBUTIONS}

Conception and design: K.J., J.H.H. Acquisition of data: K.J., J.P., J.H.J., J.C.L., J.K. Analysis: K.J., J.H.H. Interpretation of the data and drafting of the article: K.J., J.H.H. Critical revision of the article for important intellectual content: J.P., J.H.J., J.C.L., J.K., J.H.H. Final approval of the article: all authors.

\section{ORCID}

Kwangrok Jung https://orcid.org/0000-0002-2178-548X Jaewoo Park https://orcid.org/0000-0002-7107-3624 Jae Hyup Jung https://orcid.org/0000-0002-6425-9682 Jong-Chan Lee https://orcid.org/0000-0001-6590-2353 Jaihwan Kim https://orcid.org/0000-0003-0693-1415 Jin-Hyeok Hwang https://orcid.org/0000-0002-5643-8461

\section{REFERENCES}

1. Banales JM, Cardinale V, Carpino G, et al. Expert consensus document: cholangiocarcinoma: current knowledge and future perspectives consensus statement from the European Network for the Study of Cholangiocarcinoma (ENS-CCA). Nat Rev Gastroenterol Hepatol 2016;13:261-280.

2. Saha SK, Zhu AX, Fuchs CS, Brooks GA. Forty-year trends in cholangiocarcinoma incidence in the U.S.: intrahepatic disease on the rise. Oncologist 2016;21:594-599.

3. Florio AA, Ferlay J, Znaor A, et al. Global trends in intrahepatic and extrahepatic cholangiocarcinoma incidence from 1993 to 2012. Cancer 2020;126:2666-2678.

4. Sung H, Ferlay J, Siegel RL, et al. Global Cancer Statistics 2020: GLOBOCAN estimates of incidence and mortality worldwide for 36 cancers in 185 countries. CA Cancer J Clin 2021;71:209-249.

5. Siegel RL, Miller KD, Fuchs HE, Jemal A. Cancer statistics, 2021. CA Cancer J Clin 2021;71:7-33.

6. DeOliveira ML, Cunningham SC, Cameron JL, et al. Cholangiocarcinoma: thirty-one-year experience with $564 \mathrm{pa}$ tients at a single institution. Ann Surg 2007;245:755-762.

7. Hyder O, Marques H, Pulitano C, et al. A nomogram to predict long-term survival after resection for intrahepatic cholangiocarcinoma: an Eastern and Western experience. JAMA Surg 2014;149:432-438.

8. Komaya K, Ebata T, Shirai K, et al. Recurrence after resection with curative intent for distal cholangiocarcinoma. $\mathrm{Br} \mathrm{J}$ Surg 2017;104:426-433.

9. Jeong S, Cheng Q, Huang L, et al. Risk stratification system to predict recurrence of intrahepatic cholangiocarcinoma 
after hepatic resection. BMC Cancer 2017;17:464.

10. Komaya K, Ebata T, Yokoyama Y, et al. Recurrence after curative-intent resection of perihilar cholangiocarcinoma: analysis of a large cohort with a close postoperative followup approach. Surgery 2018;163:732-738.

11. Valle J, Wasan H, Palmer DH, et al. Cisplatin plus gemcitabine versus gemcitabine for biliary tract cancer. N Engl J Med 2010;362:1273-1281.

12. Valle JW, Furuse J, Jitlal M, et al. Cisplatin and gemcitabine for advanced biliary tract cancer: a meta-analysis of two randomised trials. Ann Oncol 2014;25:391-398.

13. Kim BJ, Hyung J, Yoo C, et al. Prognostic factors in patients with advanced biliary tract cancer treated with first-line gemcitabine plus cisplatin: retrospective analysis of 740 patients. Cancer Chemother Pharmacol 2017;80:209-215.

14. Sahai V, Catalano PJ, Zalupski MM, et al. Nab-paclitaxel and gemcitabine as first-line treatment of advanced or metastatic cholangiocarcinoma: a phase 2 clinical trial. JAMA Oncol 2018;4:1707-1712.

15. Sakai D, Kanai M, Kobayashi S, et al. Randomized phase III study of gemcitabine, cisplatin plus S-1 (GCS) versus gemcitabine, cisplatin (GC) for advanced biliary tract cancer (KHBO1401-MITSUBA). Ann Oncol 2018;29:viii205.

16. Morizane C, Okusaka T, Mizusawa J, et al. Combination gemcitabine plus S-1 versus gemcitabine plus cisplatin for advanced/recurrent biliary tract cancer: the FUGA-BT (JCOG1113) randomized phase III clinical trial. Ann Oncol 2019;30:1950-1958.

17. Phelip JM, Desrame J, Edeline J, et al. Modified FOLFIRINOX versus CISGEM as first-line chemotherapy for advanced biliary tract cancer: results of AMEBICA PRODIGE 38 randomized phase II trial. Ann Oncol 2020;31:S260-S261.
18. Shroff RT, Javle MM, Xiao L, et al. Gemcitabine, cisplatin, and nab-paclitaxel for the treatment of advanced biliary tract cancers: a phase 2 clinical trial. JAMA Oncol 2019;5:824830.

19. Bergquist JR, Ivanics T, Storlie CB, et al. Implications of CA19-9 elevation for survival, staging, and treatment sequencing in intrahepatic cholangiocarcinoma: a national cohort analysis. J Surg Oncol 2016;114:475-482.

20. Tella SH, Kommalapati A, Yadav S, et al. Novel staging system using carbohydrate antigen (CA) 19-9 in extra-hepatic cholangiocarcinoma and its implications on overall survival. Eur J Surg Oncol 2020;46:789-795.

21. Lamarca A, Ross P, Wasan HS, et al. Advanced intrahepatic cholangiocarcinoma: post hoc analysis of the ABC-01, -02, and -03 clinical trials. J Natl Cancer Inst 20201;112:200-210.

22. McNamara MG, Lopes A, Wasan H, et al. Landmark survival analysis and impact of anatomic site of origin in prospective clinical trials of biliary tract cancer. J Hepatol 2020;73:11091117.

23. Nakamura H, Arai Y, Totoki Y, et al. Genomic spectra of biliary tract cancer. Nat Genet 2015;47:1003-1010.

24. Javle M, Bekaii-Saab T, Jain A, et al. Biliary cancer: utility of next-generation sequencing for clinical management. Cancer 2016;122:3838-3847.

25. Wardell CP, Fujita M, Yamada T, et al. Genomic characterization of biliary tract cancers identifies driver genes and predisposing mutations. J Hepatol 2018;68:959-969.

26. Casadio M, Biancaniello F, Overi D, et al. Molecular landscape and therapeutic strategies in cholangiocarcinoma: an integrated translational approach towards precision medicine. Int J Mol Sci 2021;22:5613. 